\title{
Hysteresis and metastability of Bose-Einstein condensed clouds of atoms confined in ring potentials
}

\author{
A. Roussou ${ }^{1}$, G. D. Tsibidis ${ }^{2}$, J. Smyrnakis ${ }^{3}$, M. Magiropoulos ${ }^{3}$, \\ Nikolaos K. Efremidis ${ }^{1}$, A. D. Jackson ${ }^{4}$, and G. M. Kavoulakis ${ }^{3}$ \\ ${ }^{1}$ Department of Applied Mathematics, University of Crete, GR-71004, Heraklion, Greece \\ ${ }^{2}$ Institute of Electronic Structure and Laser (IESL), \\ Foundation for Research and Technology (FORTH), \\ N. Plastira 100, Vassilika Vouton, 70013, Heraklion, Crete, Greece \\ ${ }^{3}$ Technological Education Institute of Crete, P.O. Box 1939, GR-71004, Heraklion, Greece \\ ${ }^{4}$ The Niels Bohr Institute, and Niels Bohr International Academy, Blegdamsvej 17, Copenhagen Ø, Denmark
}

(Dated: June 18, 2021)

\begin{abstract}
We consider a Bose-Einstein condensed cloud of atoms which rotate in a toroidal/annular potential. Assuming one-dimensional motion, we evaluate the critical frequencies associated with the effect of hysteresis and the critical coupling for stability of the persistent currents. We perform these calculations using both the mean-field approximation and the method of numerical diagonalization of the many-body Hamiltonian which includes corrections due to the finiteness of the atom number.
\end{abstract}

PACS numbers: 05.30.Jp, 03.75.Lm

\section{INTRODUCTION}

Numerous experiments on cold atomic gases have been performed in recent years in topologically nontrivial trapping potentials, namely in annular/toroidal traps [1 1 ] $]$. These experiments have focused primarily on the phenomenon of the metastability of the current-carrying states.

Recently, the phenomenon of hysteresis has also been investigated in an annular potential [9]. In this experiment a Bose-Einstein condensate of sodium atoms that was initially at rest was stirred, and as the rotational frequency of the stirring potential increased, the cloud was observed to make a transition to a state with one unit of circulation at a critical frequency, $\Omega_{1}$. On the other hand, in the reverse process (i.e., starting with the gas having one unit of circulation and decreasing the frequency of the stirrer) the system was observed to return to the state with zero circulation at a different critical frequency, $\Omega_{2}$, which is a clear indication of hysteresis.

Motivated by the above experiments, we consider here the phenomenon of hysteresis in a Bose-Einstein condensed gas of atoms confined in a ring potential 10 15] as well as the stability of persistent currents [16, 17]. One of the main results of our study is the effect of the finiteness of the atom number on the phenomenon of hysteresis and on the stability of the persistent currents. To attack this problem we use the method of diagonalization of the many-body Hamiltonian. Contrary to the mean-field approximation - which makes the implicit assumption of a large particle number - the diagonalization approach includes corrections due to a finite number of atoms. In addition, it avoids the assumption of a simple product state for the many-body wavefunction that is central to the mean-field approach. As a result, this approach captures correlations that are built when the atom number is very low or when the diluteness condition is violated.

We stress that in various recent experiments it has be- come possible to trap and detect very small numbers of atoms, which can even be of order unity see, e.g., Ref. [18]. Indeed, there appears to be a more general tendency in the field of cold atoms to move towards the study of small systems. Interestingly, the vast majority of the theoretical studies which have been performed on the superfluid properties of cold atomic gases and on the phenomenon of hysteresis assume the opposite limit of large particle numbers, since they are based on the mean-field Gross-Pitaevskii approximation. As a result, very little is known about the effect of the finiteness of systems with a small number of atoms.

In the following we first present our model in Sec. II and comment on the phenomena of hysteresis and of metastability. Then, we evaluate in Sec. III the critical frequencies associated with the phenomenon of hysteresis within the mean-field approximation. In Sec. IV we go beyond the mean-field approximation to consider corrections of order $1 / N$ (and lower) due to the finiteness of the atom number $N$. In Sec. V we investigate the same question regarding the critical coupling for metastability and the matrix element for the decay rate of persistent currents in a small system. In Sec. VI we make contact with recent experiments on the phenomenon of hysteresis and of metastability, and finally in Sec. VII we present our conclusions.

\section{MODEL AND GENERAL CONSIDERATIONS}

In the present study we assume one-dimensional motion of bosonic atoms under periodic boundary conditions, as in a ring potential. This model is expected to be valid in an annular/toroidal trap as long as the interaction energy is much smaller than the energy of the trapping potential in the transverse direction.

If $c_{m}$ and $c_{m}^{\dagger}$ are annihilation and creation operators of 
an atom with angular momentum $m \hbar$, the Hamiltonian has the form

$$
\hat{H}=\frac{\hbar^{2}}{2 M R^{2}} \sum_{m} m^{2} c_{m}^{\dagger} c_{m}+\frac{U}{2} \sum_{m, n, l, k} c_{m}^{\dagger} c_{n}^{\dagger} c_{k} c_{l} \delta_{m+n, k+l} .
$$

Here $M$ is the atom mass, $R$ is the mean radius of the torus/annulus, $S$ is its cross section (in the transverse direction), with $R \gg \sqrt{S}$, and $U=2 \hbar^{2} a /(M R S)$ is the matrix element for elastic s-wave atom-atom collisions, with a scattering length $a$.

In analysing the phenomenon of hysteresis and of the metastability of superflow [16, 17], the main feature to be considered is the dispersion relation [10 15], i.e., the energy of the system as a function of the angular momentum. Let $E(\ell)$ denote the total energy where $\ell \hbar \equiv L \hbar / N$ is the angular momentum per atom and $L \hbar$ is the total angular momentum. According to Bloch's theorem [10] $E(\ell)$ consists of a periodic part plus a quadratic part which comes from the motion of the center of mass. Thus, one needs consider only $0 \leq L \leq N(0 \leq \ell \leq 1)$; the remainder of the spectrum follows trivially as a consequence of Bloch's theorem.

In the absence of interactions $E(\ell)$ consists of straight lines. In the intervals $q \leq \ell \leq q+1$ where $q$ is an integer, $E(\ell) / N=(2 q+1)|\ell| \hbar^{2} /\left(2 M R^{2}\right)$ (in what follows below we assume for simplicity that $\ell \geq 0$ ). Obviously, at the end points of each interval the first derivative of $E(\ell)$ is discontinuous. In the presence of repulsive/attractive interactions these discontinuities remain, while the curvature is negative/positive, respectively. Figure 1 shows a schematic picture of the dispersion relation $E(\ell)$ for the repulsive interactions which we consider here. Such a spectrum will give rise to hysteresis. If one goes to the rotating frame and considers $E_{\text {rot }}(\ell) / N=E(\ell) / N-\ell \hbar \Omega$, there are competing local minima as the rotational frequency of the trap $\Omega$ is varied. These competing minima give rise to discontinuous transitions and thus to hysteresis. The two critical frequencies $\Omega_{1}$ and $\Omega_{2}$ of the hysteresis loop correspond to the value of the slope of the dispersion relation $E(\ell)$ for $\ell \rightarrow 0^{+}$and $\ell \rightarrow 1^{-}$, respectively. The effect of hysteresis is thus a generic feature of this problem. On the other hand, for an effective attraction between the atoms, hysteresis is absent, since the curvature of $E(\ell)$ is positive, and thus there are no discontinuous transitions as the rotational frequency of the trap is varied.

It is convenient to write (in the interval $0 \leq \ell \leq 1$ ) the total energy per particle $E(\ell) / N$ as [19]

$$
\frac{E(\ell)}{N}=\frac{\hbar^{2}}{2 M R^{2}} \ell+e(\ell)
$$

In the case of the non-interacting problem the first term on the right gives the kinetic energy, and $e(\ell)$ vanishes. Due to Bloch's theorem, $e(\ell)$ is symmetric around $\ell=$ $1 / 2$ and a periodic function with a period equal to unity.
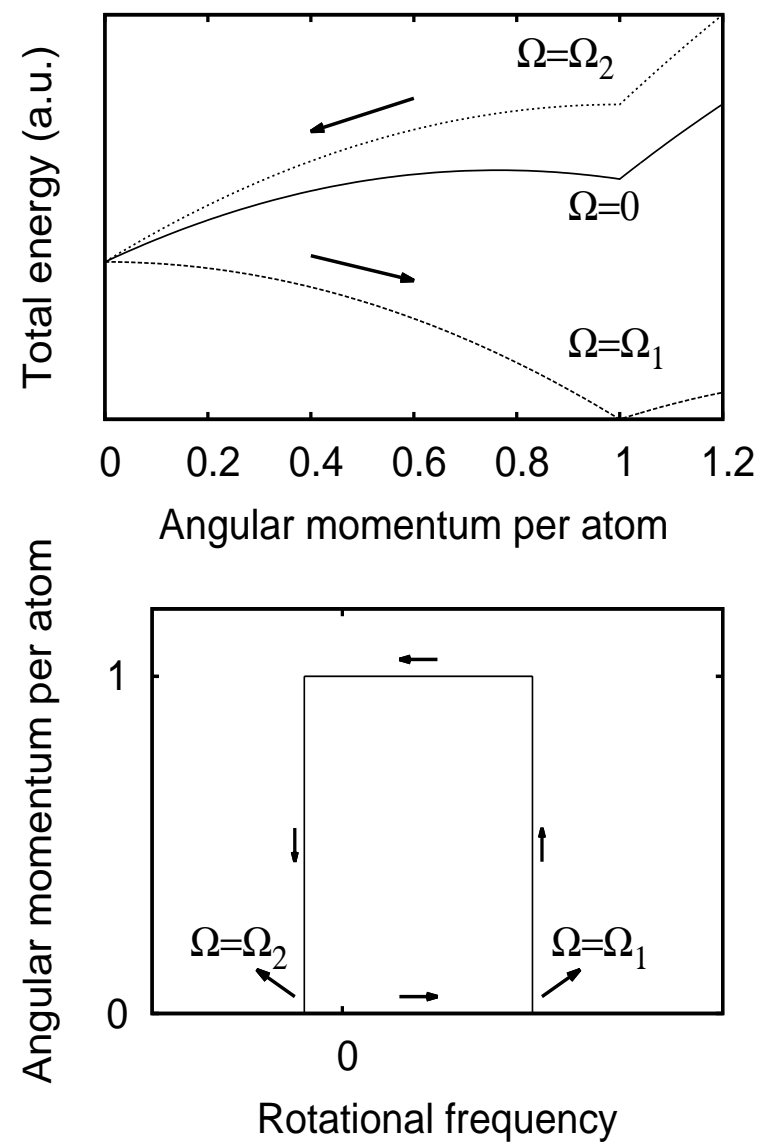

FIG. 1: Schematic plots showing the energy or, equivalently, the dispersion relation (higher), and the corresponding hysteresis loop (lower). In the dispersion relation we show the energy in the lab frame, $E(\ell)$, (middle curve) as well as in the rotating frame, $E_{\text {rot }}(\ell) / N=E(\ell) / N-\ell \hbar \Omega$, for the two critical frequencies $\Omega_{1}$ and $\Omega_{2}$ for which the slope of $E_{\text {rot }}$ vanishes for $\ell \rightarrow 0^{+}$and $\ell \rightarrow 1^{-}$, respectively. The arrows in the upper plot indicate the instability that results from the disappearance of the energy barrier; in the lower plot they indicate the hysteresis loop as the rotational frequency varies.

Expanding $e(\ell)$ for $\ell \rightarrow 0^{+}, e(\ell)=e(0)+\varepsilon \ell+\mathcal{O}\left(\ell^{2}\right)$. This implies that the slope of the dispersion relation for $\ell \rightarrow 0^{+}$is $\hbar^{2} /\left(2 M R^{2}\right)+\varepsilon$. On the other hand, for $\ell \rightarrow 1^{-}$, $e(\ell)=e(0)+\varepsilon(1-\ell)+\mathcal{O}(1-\ell)^{2}$, and thus the slope of the dispersion relation for $\ell \rightarrow 1^{-}$is $\hbar^{2} /\left(2 M R^{2}\right)-\varepsilon$. In the hysteresis loop it is precisely these slopes that determine the two critical frequencies, $\hbar \Omega_{1}=\hbar^{2} /\left(2 M R^{2}\right)+\varepsilon$ and $\hbar \Omega_{2}=\hbar^{2} /\left(2 M R^{2}\right)-\varepsilon$ as seen in the schematic plot of Fig. 1.

Therefore, it is crucial to determine the value of $\varepsilon$. Interestingly, the difference $\hbar\left(\Omega_{1}-\Omega_{2}\right)$ is equal to $2 \varepsilon$. Furthermore, the sign of $\Omega_{2}$ determines the stability of persistent currents. Specifically, the condition $\Omega_{2}=0$ represents the critical value of the coupling for metastability of the currents, and metastability will be present if $\Omega_{2}<0$. 


\section{HYSTERESIS IN THE MEAN-FIELD APPROXIMATION}

We begin with the mean-field approximation and consider the limit $\ell \rightarrow 1^{-}$. One can construct a Taylor-series expansion of the energy as a function of the small parameter $1-\ell$. Since we are interested in the slope of the dispersion relation, we only need the linear term in the expansion for the energy. To get that, it suffices to consider only the dominant state in the order parameter $\Psi$, which is $\phi_{1}$, with $\phi_{m}(\theta)=e^{i m \theta} / \sqrt{2 \pi}$ as well as the neighbouring modes $\phi_{0}$ and $\phi_{2}$. This is due to the fact that there is a cross term in the energy that comes from the scattering of two atoms with $m=1$ resulting an atom with $m=0$ and another on with $m=2$. This term can be negative and thus lowers the energy [20]. Therefore, we write the order parameter as

$$
\Psi=c_{0} \phi_{0}+c_{1} \phi_{1}+c_{2} \phi_{2},
$$

where the coefficients are real variational parameters and also $\left|c_{1}\right|$ is of order unity, while $\left|c_{0}\right|$ and $\left|c_{2}\right|$ are both of order $1-\ell$. We stress that a completely analogous calculation holds for $\ell \rightarrow 0$, in which case one should assume that $\Psi=c_{-1} \phi_{-1}+c_{0} \phi_{0}+c_{1} \phi_{1}$. We should also mention that one may work more generally with the three states $\phi_{1-\kappa}, \phi_{1}$ and $\phi_{1+\kappa}$, with $\kappa=2,3, \ldots$, however the fact that the kinetic energy of the states $\phi_{m}$ scales as $m^{2}$ necessarily implies that $\kappa=1$.

The coefficients appearing in Eq. (3) must satisfy the normalization condition, $c_{0}^{2}+c_{1}^{2}+c_{2}^{2}=1$, and the constraint of fixed angular momentum, $c_{1}^{2}+2 c_{2}^{2}=\ell$ or $c_{0}^{2}-c_{2}^{2}=1-\ell$. The expectation value of the energy per particle in the above state is

$$
\begin{aligned}
\frac{E}{N \epsilon}= & c_{1}^{2}+4 c_{2}^{2}+\frac{\gamma}{2}\left(c_{0}^{4}+c_{1}^{4}+c_{2}^{4}\right. \\
& \left.+4 c_{0}^{2} c_{1}^{2}+4 c_{1}^{2} c_{2}^{2}+4 c_{0}^{2} c_{2}^{2}-4\left|c_{0}\right| c_{1}^{2}\left|c_{2}\right|\right),
\end{aligned}
$$

where $c_{0}$ and $c_{2}$ have been assumed to have opposite signs in order to minimize the energy. Here, $\gamma / 2=N U /(2 \epsilon)=$ $2 \mathrm{NaR} / \mathrm{S}$ is the ratio between the interaction energy of the gas with a homogeneous density distribution and the kinetic energy $\epsilon \equiv \hbar^{2} /\left(2 M R^{2}\right)$. After linearisation, the above expression may also be written as

$$
\frac{E}{N \epsilon}-\frac{\gamma}{2} \approx \ell+2 c_{2}^{2}+\gamma\left(\left|c_{0}\right|-\left|c_{2}\right|\right)^{2} .
$$

Writing $c_{0}=\sqrt{1-\ell} \cosh \theta, c_{2}=\sqrt{1-\ell} \sinh \theta$, the value of $\theta$ that minimizes the energy is $\theta_{0}=(1 / 4) \ln (2 \gamma+1)$. Therefore, the minimized energy is

$$
\frac{E}{N \epsilon}-\frac{\gamma}{2} \approx \ell+[\sqrt{2 \gamma+1}-1](1-\ell) .
$$

The derived value of $\varepsilon$ is thus $\varepsilon / \epsilon=\sqrt{2 \gamma+1}-1$ and therefore

$$
\Omega_{1} / \omega=\sqrt{2 \gamma+1}
$$

while

$$
\Omega_{2} / \omega=2-\sqrt{2 \gamma+1},
$$

where $\omega=\epsilon / \hbar$. We note here that $\Omega_{2}$ will vanish if $\gamma=3 / 2$. This is the well-known result for the stability of persistent currents in a single-component gas, see, e.g., Ref. [21].

One can generalize the above results (using Bloch's theorem) in the interval $q \leq \ell \leq q+1$, where

$$
\Omega_{1} / \omega=2 q+\sqrt{2 \gamma+1},
$$

and

$$
\Omega_{2} / \omega=2(q+1)-\sqrt{2 \gamma+1} .
$$

From the last equation it follows trivially that the critical value of the coupling for stability of persistent currents (for $\ell=q+1)$ is $\gamma=(2 q+1)(2 q+3) / 2$, as Bloch's theorem implies.

\section{HYSTERESIS BEYOND THE MEAN-FIELD APPROXIMATION}

We now examine the same problem beyond the meanfield approximation. To do this, we use the method of diagonalization of the many-body Hamiltonian. To get some insight, we start with the truncated space containing the single-particle states $\phi_{0}, \phi_{1}$, and $\phi_{2}$ [i.e., the states used in Eq. (3)]. The eigenstates may be written in the form

$$
\left|\Psi_{n}\right\rangle=\sum_{p} c_{p}^{n}|p\rangle,
$$

where $n=0,1,2, \ldots$ denotes the excited state with index $n$. Here the states $|p\rangle$ are defined as $\left|0^{p}, 1^{N-2 p}, 2^{p}\right\rangle$, where the notation $\left|0^{N_{0}}, 1^{N_{1}}, 2^{N_{2}}\right\rangle$ indicates that $N_{0}$ atoms occupy the state $\phi_{0}$, etc. Clearly, the states $|p\rangle$ are eigenstates of the number operator and of the angular momentum for a system of $N$ atoms with angular momentum $L=N$. Again, one can work more generally with the three states $\phi_{1-\kappa}, \phi_{1}$ and $\phi_{1+\kappa}$, with $\kappa=2,3, \ldots$, however the corresponding problem becomes block diagonal, with the triplet of the states with $\kappa=1$ giving the slope we are looking for [21].

One can diagonalize the Hamiltonian in this truncated space using the Bogoliubov transformation to obtain the eigenvalues $\mathcal{E}_{n}(L)$, which are

$$
\begin{aligned}
\mathcal{E}_{n}(L=N) / \epsilon & -\gamma(N-1) / 2= \\
N & -(\gamma+1)+\sqrt{2 \gamma+1}(1+2 n) .
\end{aligned}
$$

Considering the states $\left|p^{\prime}\right\rangle=\left|0^{p+1}, 1^{p-2 m}, 2^{p-1}\right\rangle$ with $N$ atoms and $L=N-2$ units of angular momentum, one can follow the same procedure as before to find that

$$
\begin{aligned}
& \mathcal{E}_{n}(L=N-2) / \epsilon-\gamma(N-1) / 2= \\
& N-4-(\gamma+1)+\sqrt{2 \gamma+1}(3+2 n) .
\end{aligned}
$$




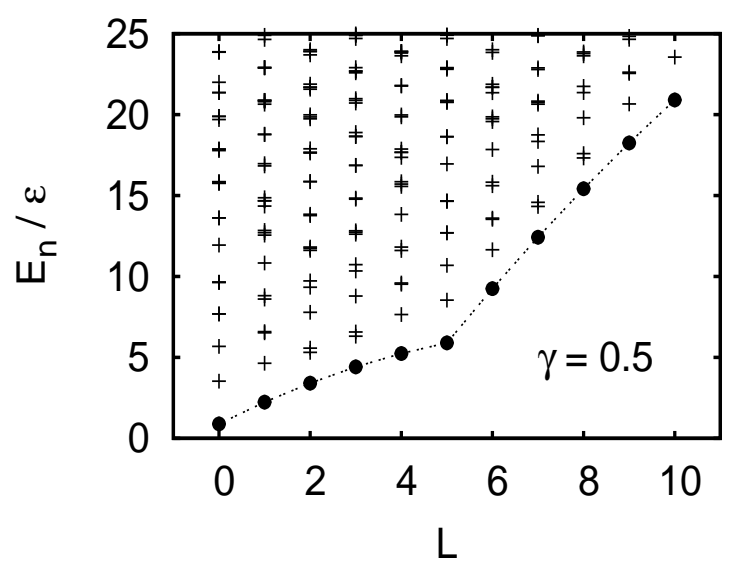

FIG. 2: The lowest eigenvalues $\mathcal{E}_{n}$ of the Hamiltonian for $N=5$ atoms as a function of $L$. Th calculation was performed for $\gamma=N U / \epsilon=0.5$, and $m_{\max }=4$.

From the lowest eigenvalues of each of the last two equations it follows that $\Omega_{2} / \omega=2-\sqrt{2 \gamma+1}$, in agreement with the result of the mean-field approximation, Eq. (8).

The approach considered above has assumed that $N$ is $\gg 1$, while the expectation value of $m$ [in Eq. (11)] is of order unity. To find the finite- $N$ corrections for the critical values of $\Omega_{1}$ and $\Omega_{2}$, we have diagonalized the many-body Hamiltonian numerically without making any approximations beyond the truncation to some set of single-particle states $\phi_{m}$ with $-m_{\max } \leq m \leq m_{\max }$. Figure 2 shows the result of such a calculation for $N=5$ atoms, $0 \leq L \leq 10, \gamma=N U / \epsilon=0.5$, and $m_{\max }=4$, where we plot a few eigenvalues for each value of $L$. The dispersion relation satisfies Bloch's theorem. The fact that the form of this figure is the same as that of the schematic plot of Fig. 1 indicates the presence of hysteresis. We stress that for the small values of $N$ that we consider here one can easily reach the Tonks-Girardeau limit. In this limit $\gamma$ is at least of order $N^{2}$. Thus, in order for the mean-field approximation to be valid, $\gamma$ has to be much less than $N^{2}$.

Having diagonalized the Hamiltonian, we extract the slope of the dispersion relation from the difference $\mathcal{E}_{0}(L=$ $1)-\mathcal{E}_{0}(L=0)$ to determine $\Omega_{1}$. Finally, by varying the atom number, $2 \leq N \leq 5$ we find that $\Omega_{1}$ can be approximated as

$$
\Omega_{1} / \omega \approx 1.0953-0.8782 / N-0.7513 / N^{2},
$$

for $\gamma=0.1$. A subtle point in this calculation is the fact that the interactions strength increases with increasing $N$. This results in a greater depletion of the condensate. Thus, in order to extract the critical frequencies associated with the hysteresis, we keep $\gamma$ fixed or equivalently allow $U$ to scale like $1 / N$.

In obtaining Eq. (14) $m_{\max }$ was set equal to 5. Clearly, $m_{\text {max }}$ must be sufficiently large so that the fitting parameters have saturated. The differences in these parameters due to changing $m_{\max }=4$ to $m_{\max }=5$ are in the seventh, third, and second significant figures respectively. The value of the leading term is remarkably close to the value of $\sqrt{1+2 \gamma} \approx 1.09544$ found in Eq. (7), which is the asymptotic value of $\Omega_{1}$ for $N \rightarrow \infty$. Similar calculations for $\gamma=1$ yield

$$
\Omega_{1} / \omega \approx 1.7453-0.6101 / N-0.1353 / N^{2} .
$$

Although the leading term is still reasonably close to $\sqrt{1+2 \gamma} \approx 1.73205$, the agreement is materially worse. This is presumably because of the larger depletion of the condensate due to the stronger interaction.

One general observation that emerges from the above analysis is that the effect of the finiteness of the system and of the correlations, captured by the method of diagonalization, is to decrease the value of $\Omega_{1}$ from its asymptotic value (and thus to increase the value of $\Omega_{2}$ ). We comment on this observation in the following section.

Last but not least, we mention that the value of the angular momentum for which the winding number of the order parameter changes is exactly $\ell=1 / 2$. In the equivalent language of solitary waves [23] the lowest-energy state with this value of the angular momentum corresponds to a "dark" solitary wave (i.e., a solitary wave with a node) which, although dark, still has a finite propagation velocity due to the finiteness of the ring [22, 23]. Assuming without loss of generality that the center of the solitary wave is located at $\theta=\pi$, the real part of the order parameter has a fixed sign. Its minimum value (at $\theta=\pi)$ vanishes as $\ell \rightarrow(1 / 2)^{-}$. The imaginary part of the order parameter has sinusoidal behaviour and vanishes at $\theta=0, \pi$, and $2 \pi$. This necessarily implies that the net phase change is zero. On the other hand, for $\ell \rightarrow(1 / 2)^{+}$, the minimum value of the real part of the order parameter, which remains $\theta=\pi$, is negative and approaches zero from below. This tiny change in the minimum value of the real part of the order parameter from slightly positive to slightly negative is sufficient to change the winding number of the phase. We stress that this tiny change can be described perturbatively and, although there is a violent rearrangement of the phase of the order parameter, this rearrangement can in no way prevent hysteresis.

\section{METASTABILITY OF PERSISTENT CURRENTS IN A SMALL SYSTEM}

The dispersion relation can develop an energy barrier for sufficiently strong and repulsive interatomic interactions which separates the state with $L=N$ from the state with $L=0$ [16]. While $\mathcal{E}_{0}(L=N)$ will always have a higher energy than $\mathcal{E}_{0}(L=0)$ [in fact, $\left.\mathcal{E}_{0}(L=N)-\mathcal{E}_{0}(L=0)=N \epsilon\right]$, the state with $L=N$ is then metastable. As a result, if the system is prepared in the state $L=N$, it will require an exponentially long time for the system to decay since this process must occur via quantum tunnelling. Furthermore, the energy and 
the angular momentum of the gas must be dissipated by small non-uniformities in the trapping potential.

In this section we investigate two different questions. The first is the critical value of the coupling required for the system to develop an energy barrier with particular concern for finite- $N$ effects. The second question is how the matrix element of a symmetry-breaking singleparticle operator $\Delta V$, that can connect the two eigenstates of lowest energy, $|L=N\rangle$ and $|L=0\rangle$, depends on the atom number $N$ (for reasons that we explain below).

Starting with the first question, according to Eq. (8) the critical value of $\gamma$ for the existence of a local minimum for $\ell \rightarrow 1^{-}$is $\gamma_{\text {cr }}=3 / 2$. This is an asymptotic result, which does not include finite- $N$ corrections. To find these corrections, we choose a fixed value of the atom number $N$ and identify the critical value of $U, U_{\text {cr }}$, which gives a zero slope in the dispersion relation for $\ell \rightarrow 1^{-}$, i.e., $\mathcal{E}_{0}(L=N)=\mathcal{E}_{0}(L=N-1)$. The result of this calculation is given in Fig. 3 where we plot the number of atoms on the $x$ axis and the product $N U_{\mathrm{cr}} \equiv \gamma_{\mathrm{cr}}$ on the $y$ axis, for $m_{\max }=4$. These results can be fit as

$$
\begin{aligned}
\gamma_{\mathrm{cr}} \approx 1.5106+0.6020 / N+8.2820 / N^{2}- & 34.8262 / N^{3} \\
& +73.3879 / N^{4}(16)
\end{aligned}
$$

The small deviation of the asymptotic value of $\gamma_{\mathrm{cr}}$ in the above expression from the expected value of $3 / 2$ is presumably due to the truncation, $m_{\max }=4$, the limited number of atoms we have considered, $N \leq 10$, and correlations which are absent in the calculation within the mean-field approximation. Interestingly, as seen from Fig. 3, the value of $\gamma_{\mathrm{cr}}$ for a finite number of atoms is higher than $3 / 2$. Since this is determined by the slope $\mathcal{E}_{0}(L=N)-\mathcal{E}_{0}(L=N-1)$, we conclude that the correlations which are captured within the present approach (but are absent within the mean-field approximation) lower the energy of the state with $L=N-1$ more than that of the state with $L=N$. Thus, a higher value of $\gamma$ is necessary to stabilize the currents in the state with $L=N$. The same mechanism which increases $\gamma_{\text {cr }}$ is also responsible for the decrease (increase) of $\Omega_{1}\left(\Omega_{2}\right)$ found in the previous section.

We turn now to the second question regarding the decay rate of the persistent current. In order for the energy barrier (which develops for sufficiently strong interatomic interactions) to prevent the decay of the currents and render them metastable with an exponentially long decay time, the matrix element of any symmetry-breaking single-particle operator $\Delta V$ connecting the states $\mid L=$ $N\rangle$ and $|L=0\rangle$ must be vanishingly small [24]. Otherwise the presence of the energy barrier becomes irrelevant and the currents will decay.

To investigate this problem, we consider a singleparticle operator $\Delta V=V_{0} \sum_{i=1}^{N} \delta\left(\theta_{i}\right)$, which is a sum of delta function potentials intended to mimic irregularities in the trap [12]. This potential breaks the axial symmetry of the Hamiltonian and induces transitions between the two states $|L=N\rangle$ and $|L=0\rangle$. We thus evaluate the matrix element $\langle L=N|\Delta V| L=0\rangle$, making use of

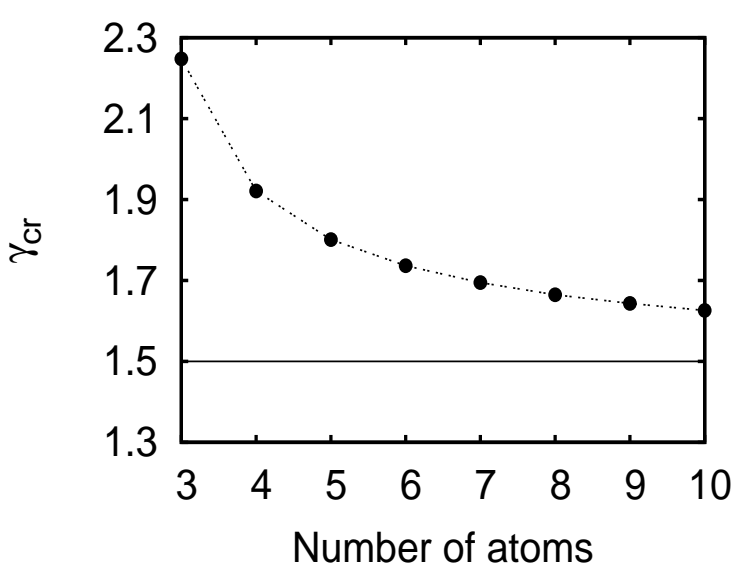

FIG. 3: The value of $\gamma_{\mathrm{cr}}$ obtained with the method of diagonalization as a function of $N$ for $m_{\max }=4$. The horizontal line shows the asymptotic value of $\gamma_{\mathrm{cr}}=3 / 2$.

the lowest-energy states $|L=0\rangle$ and $|L=N\rangle$ that we get from the diagonalization of the axially-symmetric Hamiltonian. Clearly the only terms which give a nonzero contribution to this matrix element are those that raise the angular momentum by $L=N$ units when acting on $|L=0\rangle$,

$$
\langle L=N|\Delta V| L=0\rangle=V_{0} \sum_{n}\left\langle L=N\left|a_{n} a_{n+N}^{\dagger}\right| L=0\right\rangle .
$$

In the absence of interactions, when all the atoms are in the single-particle state $\phi_{1}$ and $\phi_{0}$, respectively, this matrix element vanishes for all $N>1$. This is also the case in the mean-field approximation. To get a non-vanishing matrix element it is necessary to consider non-zero interactions that deplete the condensate and a finite number of atoms.

Figure 4 shows the value of $\left|\langle L=N|\Delta V| L=0\rangle / V_{0}\right|$ as function of $N$. Again, we keep $\gamma=g N$ fixed for the reasons stated above. Here, we have chosen $\gamma=g N=$ 0.1 , while the states $|L=0\rangle$ and $|L=N\rangle$ have been evaluated for $m_{\max }=5$. As seen from this plot, this matrix element shows an exponential decay as function of $N$.

To get an understanding of this decay we recall that the operator $\Delta V$ excites atoms, increasing their angular momentum by $N$ units. Furthermore, the amplitudes $c_{m}$ in the expression of Eq. (11) decay very rapidly with $m$, as seen in Fig. 5 for $N=50$ atoms with a rate that does not depend on $N$. This is a more general result that also holds in more extended spaces. The fact that the amplitudes of the states contributing to $|L=0\rangle$ and $\mid L=$ $N\rangle$ decrease rapidly as one moves away from $\left|0^{N}\right\rangle$ and $\left|1^{N}\right\rangle$ along with the nature of $\Delta V$, which induces singleparticle excitations by $N$ units of angular momentum, combine to make this decay matrix element extremely sensitive to $N$. 


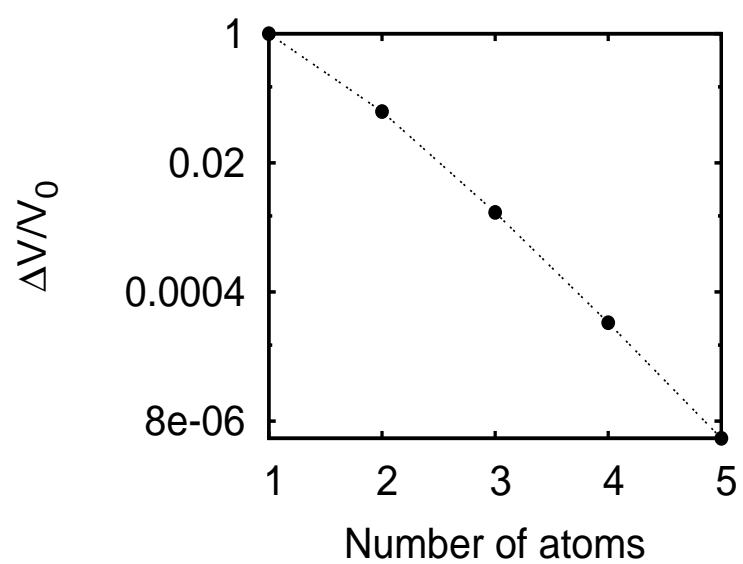

FIG. 4: The matrix element of the operator $\Delta V$ between the states with $|L=N\rangle$ and $|L=0\rangle,\left|\langle L=N|\Delta V| L=0\rangle / V_{0}\right|$, as function of the atom number $N$, for a fixed value of $\gamma=$ $g N=0.1$. Here the states $|L=0\rangle$ and $|L=N\rangle$ have been evaluated for $m_{\max }=5$.

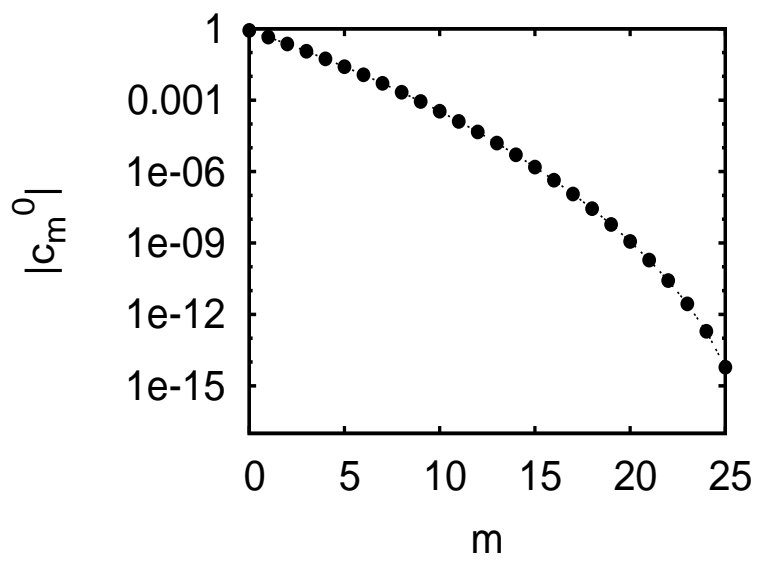

FIG. 5: The amplitudes $\left|c_{m}^{0}\right|$ which appear in Eq. (11) as function of the index $m$, for $N=50$ atoms, $L=0, \gamma=5$, and truncation within the single-particle states $\phi_{-1}, \phi_{0}$, and $\phi_{1}$.

Thus, the main result of this section is, quite generally, that a combination of sufficiently strong interatomic interactions and a finite number of atoms enhances the size of the matrix element and thus reduces the timescale that is associated with the decay rate of the persistent currents. This result may be interesting to explore experimentally in small systems with an interaction whose strength can be tuned.

\section{CONNECTION WITH THE EXPERIMENTS ON HYSTERESIS AND METASTABILITY}

In order for our assumption of one-dimensional motion to be valid, the interaction energy must be much smaller than the quantum of energy associated with the motion of the atoms in the transverse direction (or, equivalently, the coherence length must be much larger than the transverse dimensions of the annulus/torus). However, this assumption is violated under current typical conditions, and thus the motion is not quasi-one-dimensional.

For example, in the experiment of Ref. [9], where ${ }^{23} \mathrm{Na}$ atoms were used, the chemical potential is $\mu / \hbar \approx 2 \pi \times 1.7$ $\mathrm{kHz}$, while the frequencies of the annular-like trapping potential (in the transverse direction) are $\omega_{1} \approx 472 \mathrm{~Hz}$ and $\omega_{2} \approx 188 \mathrm{~Hz}$. (As a result, it has been argued that vortex-antivortex pairs form in this experiment.) Thus, it is not possible to make neither a quantitative nor a qualitative comparison of the present theory and the experiment of Ref. [9]. An investigation of this problem using a more realistic model is underway and will be described in a future publication.

If one wants nonetheless to get an estimate for the critical frequencies of hysteresis for the parameters of Ref. [9] using the present theory, it follows for a radius of $R \approx 19.5 \mu \mathrm{m}$, that $\omega=\hbar /\left(2 M R^{2}\right) \approx 3.6 \mathrm{~Hz}$. Given that $a \approx 28 \AA, N \approx 4 \times 10^{5}$, and $S=\pi a_{1} a_{2}$ with $a_{i}=\sqrt{\hbar /\left(M \omega_{i}\right)}$, i.e., $a_{1} \approx 2.42 \mu \mathrm{m}$ and $a_{2} \approx 3.83 \mu \mathrm{m}$, the dimensionless parameter $\gamma=2 \mathrm{NaR} / \mathrm{S}$ has the value $\gamma \approx 1500.0$. It then follows from Eqs. (7) and (8) that $\Omega_{1} \approx 197.2 \mathrm{~Hz}$ and $\Omega_{2} \approx-190.0 \mathrm{~Hz}$. Clearly, these large frequencies (as compared to the observed frequencies, which are on the order of $10 \mathrm{~Hz}$ ) are due to the very large value of $\gamma$, which is the ratio between the interaction energy of a homogeneous cloud with a density $n_{0}=N /(2 \pi R S)$ and the kinetic energy associated with the motion in the ring, $\hbar^{2} /\left(2 M R^{2}\right)$.

It is also interesting to make estimates for the case where the motion is quasi-one-dimensional. Consider, for example, the case where the experimental conditions are identical to those of Ref. [9] but where the number of atoms is reduced by, e.g., a factor of $4 \times 10^{4}$ to the value $N=10$. This would reduce the interaction energy to the extent that the conditions for one-dimensional motion would be fulfilled. This reduction in $N$ would also reduce the value of $\gamma$ to $\approx 0.04$. The corresponding critical frequencies would become $\Omega_{1} \approx 3.7 \mathrm{~Hz}$ and $\Omega_{2} \approx 3.5 \mathrm{~Hz}$. While the difference between $\Omega_{1}$ and $\Omega_{2}$ is small, $\approx 2 \gamma \omega$, it would still be of interest to investigate their dependence on $N$, which, according to the results of Sec. IV, is $1 / N$ to leading order.

It would also be interesting to investigate the effect of finite system size on the critical value for stability of the persistent currents in such small systems. According to the results of Sec. V, the value of $\gamma_{\mathrm{cr}}$ also scales as $1 / N$ to leading order. Last but not least, the decay time of the currents would show a much more rapid - and thus more pronounced - decrease as $N$ decreases.

\section{CONCLUSIONS}

In the present study we have investigated the phenomenon of hysteresis and of metastability in a Bose- 
Einstein condensed cloud of atoms which are confined in a ring potential. Interestingly, this problem has recently been examined experimentally [9], while many other experiments have focused on the question of persistent currents in such topologically nontrivial potentials [1 8 ].

In the phenomenon of hysteresis the main question is the evaluation of the critical frequencies. As we have shown, in a purely one-dimensional system these two frequencies are related as a consequence of Bloch's theorem. Further, we have evaluated those both within the meanfield approximation and beyond mean field (i.e., by numerical diagonalization of the many-body Hamiltonian) in order to determine finite- $N$ corrections.

We have also performed calculations of the critical coupling for the metastabiliity of superflow and of the matrix element associated with the decay rate in a finite system of atoms. As we have argued, the depletion of the condensate due to the interaction combined with the finiteness of the atom number can cause the decay rate to increase exponentially with decreasing $N$. Thus, the general tendency is that the finiteness of a system makes the supercurrents more fragile, in the sense that it increases the decay rate of the currents, and it also increases the critical coupling for metastability.

Given the recent experimental activities on the problems of hysteresis and of metastability, and also given the more general tendency in the community of cold atoms to move to small systems (i.e., systems with a small atom number $N$ ) the present results, which we believe are of theoretical interest, may will become experimentally relevant in the near future.

\section{Acknowledgments}

This project is implemented through the Operational Program "Education and Lifelong Learning", Action Archimedes III and is co-financed by the European Union (European Social Fund) and Greek national funds (National Strategic Reference Framework 2007 - 2013).
[1] S. Gupta, K. W. Murch, K. L. Moore, T. P. Purdy, and D. M. Stamper-Kurn, Phys. Rev. Lett. 95, 143201 (2005).

[2] Spencer E. Olson, Matthew L. Terraciano, Mark Bashkansky, and Fredrik K. Fatemi, Phys. Rev. A 76, 061404(R) (2007).

[3] C. Ryu, M. F. Andersen, P. Cladé, Vasant Natarajan, K. Helmerson, and W. D. Phillips, Phys. Rev. Lett. 99, 260401 (2007).

[4] B. E. Sherlock, M. Gildemeister, E. Owen, E. Nugent, and C. J. Foot, Phys. Rev. A 83, 043408 (2011).

[5] A. Ramanathan, K. C. Wright, S. R. Muniz, M. Zelan, W. T. Hill, C. J. Lobb, K. Helmerson, W. D. Phillips, and G. K. Campbell, Phys. Rev. Lett. 106, 130401 (2011).

[6] Stuart Moulder, Scott Beattie, Robert P. Smith, Naaman Tammuz, and Zoran Hadzibabic, Phys. Rev. A 86, 013629 (2012).

[7] C. Ryu, K. C. Henderson and M. G. Boshier, New J. Phys. 16, 013046 (2014).

[8] Scott Beattie, Stuart Moulder, Richard J. Fletcher, and Zoran Hadzibabic, Phys. Rev. Lett. 110, 025301 (2013).

[9] Stephen Eckel, Jeffrey G. Lee, Fred Jendrzejewski, Noel Murray, Charles W. Clark, Christopher J. Lobb,William D. Phillips, Mark Edwards, and Gretchen K. Campbell, Nature (London) 506, 200 (2014).

[10] F. Bloch, Phys. Rev. A 7, 2187 (1973).

[11] Erich J. Mueller, Phys. Rev. A 66, 063603 (2002).

[12] G. M. Kavoulakis, Y. Yu, M. Ögren, and S. M. Reimann, Europhys. Lett. 76, 215 (2006).
[13] R. Kanamoto, L. D. Carr, and M. Ueda, Phys. Rev. A 79, 063616 (2009).

[14] R. Kanamoto, L. D. Carr, and M. Ueda, Phys. Rev. A 81, 023625 (2010).

[15] Soheil Baharian and Gordon Baym, Phys. Rev. A 87, 013619 (2013).

[16] Anthony J. Leggett, Rev. Mod. Phys. 73, 307 (2003).

[17] A. I. Yakimenko, S. I. Vilchinskii, Y. M. Bidasyuk, Y. I. Kuriatnikov, K. O. Isaieva, and M. Weyrauch, e-print arXiv:1411.3490

[18] A. N. Wenz, G. Zürn, S. Murmann, I. Brouzos, T. Lompe, and S. Jochim, Science 342, 457 (2013).

[19] We stress that this expression is consistent with Bloch's theorem, since the energy may also be written as $E(\ell) / N=\left[\hbar^{2} /\left(2 M R^{2}\right)\right]\left[\ell^{2}+\left(\ell^{2}-\ell\right)\right]+e(\ell)$, where the last three terms are periodic.

[20] G. M. Kavoulakis, B. Mottelson, and C. J. Pethick, Phys. Rev. A 62, 063605 (2000).

[21] M. Ögren and G. M. Kavoulakis, J. Low Temp. Phys. 149, 176 (2007).

[22] J. Smyrnakis, M. Magiropoulos, G. M. Kavoulakis, and A. D. Jackson, Phys. Rev. A 82, 023604 (2010).

[23] A. D. Jackson, J. Smyrnakis, M. Magiropoulos, and G. M. Kavoulakis, Europh. Lett. 95, 30002 (2011).

[24] There is another way for the currents to decay, via highorder perturbation theory, however this process is also highly suppressed for weak irregularities. 